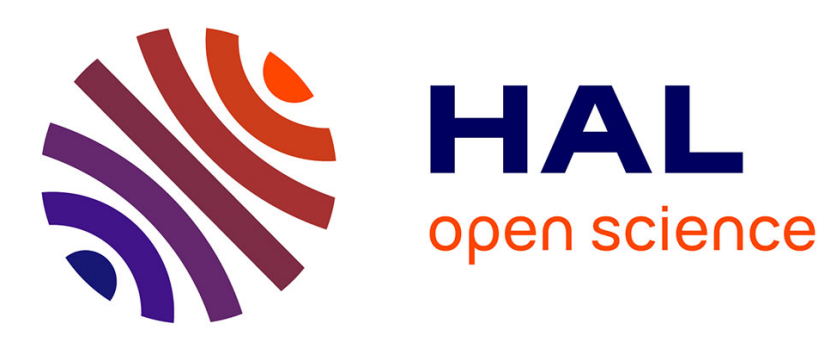

\title{
Service-oriented bi-objective robust collection-disassembly problem with equipment selection
}

\author{
Xin Liu, Feng Chu, Alexandre Dolgui, Feifeng Zheng, Ming Liu
}

\section{To cite this version:}

Xin Liu, Feng Chu, Alexandre Dolgui, Feifeng Zheng, Ming Liu. Service-oriented bi-objective robust collection-disassembly problem with equipment selection. International Journal of Production Research, 2021, 59 (6), pp.1676-1690. 10.1080/00207543.2020.1723815 . hal-02488907

\section{HAL Id: hal-02488907 https://hal.science/hal-02488907}

Submitted on 24 Mar 2021

HAL is a multi-disciplinary open access archive for the deposit and dissemination of scientific research documents, whether they are published or not. The documents may come from teaching and research institutions in France or abroad, or from public or private research centers.
L'archive ouverte pluridisciplinaire HAL, est destinée au dépôt et à la diffusion de documents scientifiques de niveau recherche, publiés ou non, émanant des établissements d'enseignement et de recherche français ou étrangers, des laboratoires publics ou privés. 


\title{
Service-oriented bi-objective robust collection-disassembly problem with handling module
}

\author{
Xin $\operatorname{Liu}^{a}$, Feng $\mathrm{Chu}^{b, c}$, Alexandre Dolgui ${ }^{d}$, Feifeng Zheng ${ }^{e}$, Ming Liu $^{a}$ \\ ${ }^{a}$ School of Economics \&3 Management, Tongji University, Shanghai, 200092, China \\ ${ }^{b}$ School of Economics \& Management, Fuzhou university, Fujian, 350108, China \\ ${ }^{c}$ IBISC, Univ Évry, University of Paris-Saclay, 91025 Évry, France \\ ${ }^{d}$ LS2N, UMR CNRS 6004, IMT Atlantique, Nantes Cedex 3, France \\ ${ }^{e}$ Glorious Sun School of Business 83 Management, Donghua University, China
}

\begin{abstract}
The collection-disassembly problem plays an important role in the reverse supply chain management. The focus of the problem is to coordinate the activities associated with the collection and the disassembly of the end-of-life (EOL) products. Most existing works consider the problem under deterministic settings. However, in practice, the demands of components of EOL products that can be reused may be uncertain, due to various factors. Besides, it is usually difficult to obtain the probability distribution of the uncertain demands, as the historical data may be inadequate. This paper investigates a stochastic collection-disassembly problem, in which handling modules of disassembly system, corresponding to different disassembly capacities, have to be selected and partial distributional information of component demands (i.e., the mean and covariance matrix of demands) is known. The objectives are to minimize the system cost and to maximize the average customer service level (i.e., the average probability of meeting each demand) simultaneously. For the problem, a novel distributionally robust bi-objective formulation is proposed. Based on the proposed model, the Monte carlo simulation and an ambiguity set, a sample average approximation (SAA) model and an approximated mixed integer programming (MIP) model are constructed. Then the two approximated formulations are solved, via the $\epsilon$-constraint framework,
\end{abstract}

Email address: mingliu@tongji.edu.cn (Ming Liu $\left.{ }^{a}\right)$ 
and compared.

Keywords: Reverse supply chain; Collection of EOL products; Service level; Bi-objective optimization; Handling module selection

\section{Introduction}

In recent decades, governments and customers have paid more attention to the sustainabile manufacturing and supply chain management. As recycling or remanufacturing End-of-Life (EOL) products can realize resource re-utilisation, saving energy and air pollutant reduction as well, thus it is getting more emphasized (Ilgin and Gupta, 2010a). Recycling EOL products mainly includes various activities: the collection, disassembly, recycling and storing materials and disposal of waste (Ilgin and Gupta, 2010b; Bentaha et al., 2014a). Among them, the collection and the disassembly of EOL products are essential and important (Habibi et al., 2017a). Existing studies usually consider the collection of EOL products and the subsequent disassembly activities separately (Mcgovern and Gupta, 2007; Agrawal and Tiwari, 2008). Habibi et al. (2017b) introduce the collection-disassembly problem and show that the joint optimization of collection and disassembly decisions can provide both economic profile and customer satisfaction improvement. As shown in Figure 1, the problem is to design vehicle routes to collect EOL products from collection centers to disassembly site, and select handling modules for the disassembly system, and then disassemble the EOL products into components, to satisfy the demands.

Related works on the collection-disassembly problem mainly focus on the deterministic settings (Habibi et al., 2017a; Habibi et al., 2017b). In practical reverse supply chain, however, the demand of components of EOL products that can be reused may be uncertain, due to the volatile market and environmental policies and seasonal trends (Özceylan and Paksoy, 2014; Tuncel et al., 2014; Ivanov et al., 2017). To our best knowledge, Habibi et al. (2019) first study the collection-disassembly problem with uncertain demands of components. They assume that the probability distribution of uncertain demands is known. However, for some products with high regeneration and replacement speed, such as the electrical and electronic equipment, the historical data may be not representative (Delage and Ye, 2010). Thus the complete probability distribution of the uncertain demands may not be well estimated. For this concern, this work considers a stochastic collection- 
disassembly problem, under partial known distributional information (i.e., the empirical mean and covariance matrix of demands).

In addition to the common target of reducing system cost, increasing customer service level (i.e., the reduction of the risk level) has always been an important goal in supply chain management (Cardona-Valds et al., 2011; Cardona-Valds et al., 2014). Customer satisfaction and service level are usually considered as an important performance indicator of supply chain (Bijvank and Vis, 2012). In this work, EOL product are first collected from collection centers to disassembly site, and then disassembled into components which are required by customers, as shown in Figure 1. Failing to fulfill the demand for the components may cause great loss for companies, such as low customer satisfaction and loyalty loss (Tiwari et al., 2010). Therefore, to provide better scientific support for the decision makers, the pursued objective is to minimize the system cost and to maximize the customer service level simultaneously.

Moreover, it is usually assumed in the literature that the disassembly line capacity is fixed (Habibi et al., 2017a; Habibi et al., 2019). However, in practice, a disassembly system possesses a set of machines and equipments (Paksoy, 2013), and different machine and equipment combinations can be considered as handling modules, corresponding to different disassembly line capacities (Li et al., 2011; Liu et al., 2019), as shown in Figure 1. Appropriately selecting handling module for each disassembly line may improve the overall performance of the reverse supply chain.

Motivated by the above observations, this work studies a stochastic biobjective collection-disassembly problem with handling module selection and partial distributional information of demands of components (i.e., the empirical mean and covariance matrix). The two objectives are to minimize the system cost and to maximize the customer service level simultaneously. The contribution of this paper mainly includes:

(1) A new stochastic bi-objective collection-disassembly problem with partial distributional information on the demands of components and handling module selection is investigated.

(2) For the problem, a distributionally robust bi-objective formulation is first proposed, to minimize the system cost and maximize the service level.

(3) Based on two approximation methods for the partial known probability 
distribution, two approximated models are developed and solved via the $\epsilon$-constraint framework.

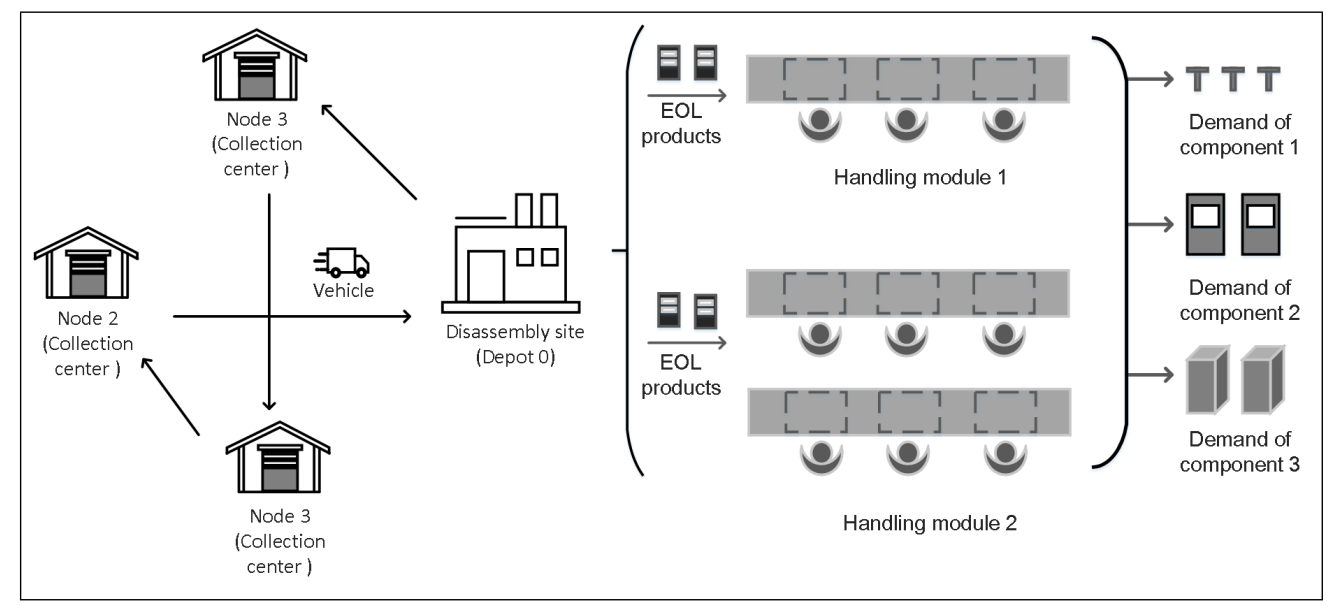

Figure 1: An illustrative example of the problem during one period

The paper is organized as follows. A brief literature review is given in Section 2. Section 3 gives the problem description and proposes a distributionally robust bi-objective formulation. In Section 4, based on two approximation methods for the partial probability distribution, two approximated models are constructed. Computational results are reported and analyzed in Section 5. Section 6 summarize this paper and suggests future research directions.

\section{Literature review}

The collection-disassembly problem is first introduced by Habibi et al. (2017a), which focuses on collecting EOL products, selecting the handling modules for the disassembly system and the subsequent disassembly. As this work considers the bi-objective collection-disassembly problem with partial distributional information of the component demands, to minimize the system cost and maximize the customer service level, we only review the most related studies in the following.

Stochastic disassembly line balancing problem has been studied by various researchers (Bentaha et al., 2012; Bentaha et al., 2013; Bentaha et al., 2014a; Bentaha et al., Bentaha et al.; Bentaha et al., 2015; Özceylan et al., 
2018). However, most existing works considering uncertain task processing times, while the studies for the uncertain demand are very few. Özceylan and Paksoy (2014) investigate a disassembly line balancing problem with uncertain demands that are described by fuzzy membership function. Tuncel et al. (2014) investigate a stochastic disassembly line balancing problem, where the demand for individual components is assumed to be stochastic. The authors develop a Monte-Carlo-based reinforcement learning technique to solve it.

The collection-disassembly problem, which focuses on designing the vehicle routes to collect EOL products and the disassembly decisions to satisfy the demands of components, has been investigated by the following works. Habibi et al. (2017a) first consider the coordination of the collection and disassembly of EOL products in the deterministic settings. They propose an integrated model for incorporating the two aspects, and they also show the coordination of collection and disassembly decisions can provide the performance improvement of the reverse supply chain, in terms of the total cost and the component demand satisfaction. The same problem under deterministic environment is further considered by Habibi et al. (2017b). A two-phase iterative heuristic is developed, to solve large-scale instances. Habibi et al. (2019) first investigate a multi-vehicle collection-disassembly problem The quality, the available EOL products and the demands are assumed to be uncertain, under known probability distributions. The objective is to minimize the expected cost. Then a two-stage stochastic programming formulation is proposed, and a two-phase iterative heuristic is developed.

Concluding, to our best knowledge, there is no research on the bi-objective collection-disassembly problem in the literature, with only partial distributional information on the uncertain demands. We broaden the realm of the collection-disassembly problem via considering (i) partial demand information, i.e., in a distributionally robust fashion, (ii) handling module selection and (iii) service level maximization.

\section{Problem description and formulation}

In this section, we first describe the problem in detail and give basic assumptions and then propose a new distributionally robust bi-objective formulation. 


\subsection{Problem description}

This work investigates a stochastic collection-disassembly problem with alternative handling modules for disassembly line. The problem consists of a set $\mathcal{T}=\{1,2, \ldots,|\mathcal{T}|\}$ of periods, and the problem during one period is shown in Figure 1. There is a set of nodes $\mathcal{N}=\{0,1,2, \ldots,|\mathcal{N}|\}$, where 0 denotes the depot and set $\mathcal{N}_{c}=\{1,2, \ldots,|\mathcal{N}|\}$ denotes the set of collection centers. In Figure 1, for example, there is a disassemble site (i.e., the depot 0) and 3 collection centers, i.e., nodes 1, 2 and 3 . In the problem:

(1) There is a set $\mathcal{K}=\{1,2, \ldots,|\mathcal{K}|\}$ of vehicles. During each period $t \in$ $\mathcal{T}$, EOL products are first collected from collection centers and then disassembled into components, and all components are included in set $\mathcal{L}=\{1,2, \ldots,|\mathcal{L}|\}$.

(2) We assume that the EOL products are regular, such as toys, daily necessities and common electronic products. The amount $S_{i t}$ of available EOL products at collection center $i \in \mathcal{N}_{c}$ during period $t \in \mathcal{T}$ is assumed to be fixed.

(3) For the disassembly system, as stated above, there is a set $\mathcal{H}$ of alternative handling modules, i.e., $\mathcal{H}=\{1,2, \ldots,|\mathcal{H}|\}$, and each handling module $h \in \mathcal{H}$ corresponds to operating cost $C_{h}^{M}$ and disassembly line capacity $V_{h}^{\text {dis }}$.

(4) The amount of component $l \in \mathcal{L}$ in each EOL product is fixed and denoted as $n_{l}$. The demand $\xi_{l t}$ of component $l \in \mathcal{L}$ during period $t \in \mathcal{T}$ is assumed to be uncertain, and all demands are represented by an uncertain vector $\boldsymbol{\xi}=\left[\xi_{1,1}, \xi_{1,2}, \ldots \xi_{1,|\mathcal{T}|}, \xi_{2,1}, \ldots, \xi_{2,|\mathcal{T}|}, \ldots, \xi_{|\mathcal{L}|,|\mathcal{T}|}\right]$. Only partial distributional knowledge of $\boldsymbol{\xi}$, i.e., the empirical mean and covariance matrix, is known.

(5) The vehicle capacity and the inventory capacity of the disassembly site (i.e., the maximum number of EOL products stored at the disassembly site) are fixed and denoted by $Q$ and $V^{i n v}$, respectively.

As stated above, the accurate probability distribution of demands, denoted by $\mathbb{P}$, is unavailable. A finite set $\mathcal{R}=\{1,2, \ldots,|\mathcal{R}|\}$ of historical samples,

i.e., $\left\{\boldsymbol{\xi}^{r}\right\}_{r=1}^{|\mathcal{R}|}$, is given. The empirical mean vector $\boldsymbol{\mu}$ and covariance matrix 
$\Gamma$ can be estimated as:

$$
\boldsymbol{\mu}=\frac{1}{|\mathcal{R}|} \sum_{r \in \mathcal{R}} \boldsymbol{\xi}_{r}, \quad \boldsymbol{\Gamma}=\frac{1}{|\mathcal{R}|} \sum_{r \in \mathcal{R}}\left(\boldsymbol{\xi}^{r}-\boldsymbol{\mu}\right)\left(\boldsymbol{\xi}^{r}-\boldsymbol{\mu}\right)^{\top},
$$

where $(\cdot)^{\top}$ denotes the transpose of a vector.

During each period, the considered problem is to (i) deploy vehicles and design the vehicle routes, to collect EOL products, (ii) select handling module for the disassembly line, (iii) determine the amount of EOL products disassembled and the inventory level at the disassemble site. The objectives are to minimize the system cost and to maximize the average customer service level (i.e., the average probability of meeting each demand) simultaneously.

\subsection{Formulation}

In this subsection, a distributionally robust bi-objective formulation for the considered problem is proposed. In the following, we first describe basic notations and decision variable, and then present the formulation.

\section{Parameters}

- $\mathcal{N}$ : Set of nodes indexed by $i, j \in\{0,1,2, \ldots,|\mathcal{N}|\}$, in which node 0 denotes the depot (i.e., the disassembly site).

- $\mathcal{N}_{c}$ : Set of collection centres indexed by $i, j \in\{1,2, \ldots,|\mathcal{N}|\}$.

- $\mathcal{L}$ : Set of components indexed by $l \in\{1,2, \ldots,|\mathcal{L}|\}$.

- $\mathcal{T}$ : Planning horizon and $\mathcal{T}=\{1,2, \ldots,|\mathcal{T}|\}$.

- $\mathcal{K}$ : Set of vehicles indexed by $k \in\{1,2, \ldots,|\mathcal{K}|\}$.

- $\mathcal{H}$ : Set of handling modules indexed by $h \in\{1,2, \ldots,|\mathcal{H}|\}$ for the disassembly line.

- $n_{l}$ : Number of component $l \in \mathcal{L}$ in an EOL product.

- $S_{i t}$ : Number of EOL products available at collection center $i \in \mathcal{N}_{c}$ during period $t \in \mathcal{T}$.

- Q: Vehicle capacity.

- $\xi_{l t}$ : Demand of component $l \in \mathcal{L}$ during period $t \in \mathcal{T}$, which is stochastic. 
- $V^{\text {inv }}$ : Inventory capacity of the disassembly site for storing EOL products.

- $V_{h}^{\text {dis }}$ : Disassembly capacity of handling module $h \in \mathcal{H}$.

- $C_{h}^{M}$ : Fixed operating cost for handling module $h \in \mathcal{H}$.

- $C^{f}$ : Fixed vehicle deploying cost.

- $c_{i j}$ : Cost for a vehicle traveling from node $i$ to $j$.

- $C^{\text {inv }}$ : Inventory cost in each time unit.

- $C^{d}$ : Unit disassembly cost.

- $\theta_{l t}$ : the weight coefficient of each individual service level (i.e., the probability of meeting demand) of component $l \in \mathcal{L}$ during period $t \in \mathcal{T}$, and $\sum_{l \in \mathcal{L}} \sum_{t \in \mathcal{T}} \theta_{l t}=1$.

\section{Decision variables}

- $z_{t}$ : Number of vehicles deployed during period $t \in \mathcal{T}$.

- $\eta_{h t}$ : Binary variable, equal to 1 if handling module $h \in \mathcal{H}$ is selected during period $t \in \mathcal{T}$.

- $x_{i j t}^{k}$ : Binary variable, equal to 1 if node $j$ is visited immediately after $i$ by vehicle $k$ during period $t \in \mathcal{T}$.

- $y_{i t}^{k}$ : The load of vehicle $k$ after visiting node $i$ during period $t$.

- $I_{t}$ : Inventory level of EOL products during period $t$.

- $P_{t}$ : Quantity of EOL products disassembled during period $t$.

A distributionally robust bi-objective formulation $[\mathbf{P} 1]$ for the problem is proposed as follows:

$$
\begin{aligned}
{[\mathbf{P} 1]: } & \\
\min f_{1} & =\left\{\sum_{t \in \mathcal{T}}\left(\sum_{h \in \mathcal{H}} C_{h}^{M} \eta_{h t}+C^{f} z_{t}+\sum_{k \in \mathcal{K}} \sum_{i, j \in \mathcal{N}, i \neq j} c_{i j} x_{i j t}^{k}+C^{i n v} I_{t}+C^{d} P_{t}\right)\right\} \\
\max f_{2} & =\left\{\sum_{l \in \mathcal{L}} \sum_{t \in \mathcal{T}} \theta_{l t} \cdot\left(\operatorname{Prob}_{\mathbb{P}}\left(n_{l} \cdot P_{t} \geq \xi_{l t}\right)\right)\right\}
\end{aligned}
$$




$$
\begin{aligned}
& \text { s.t. } \sum_{k \in \mathcal{K}} \sum_{j \in \mathcal{N}, i \neq j} x_{i j t}^{k} \leq 1, \quad \forall i \in \mathcal{N}_{c}, t \in \mathcal{T} \\
& \sum_{i \in \mathcal{N}_{c}} x_{0 i t}^{k} \leq 1, \quad \forall k \in \mathcal{K}, t \in \mathcal{T} \\
& \sum_{k \in \mathcal{K}} \sum_{i \in \mathcal{N}_{c}} x_{0 i t}^{k} \leq z_{t}, \quad \forall t \in \mathcal{T} \\
& \sum_{j \in \mathcal{N}, i \neq j} x_{i j t}^{k}=\sum_{j \in \mathcal{N}, i \neq j} x_{j i t}^{k}, \quad \forall i \in \mathcal{N}, k \in \mathcal{K}, t \in \mathcal{T} \\
& \sum_{h \in \mathcal{H}} \eta_{h t}=1, \quad \forall t \in \mathcal{T} \\
& y_{i t}^{k}+\left(Q-S_{i t}\right) \cdot x_{0 i t}^{k} \leq Q, \quad \forall i \in \mathcal{N}_{c}, k \in \mathcal{K}, t \in \mathcal{T} \\
& y_{i t}^{k}-y_{j t}^{k}+Q \cdot x_{i j t}^{k}+\left(Q-S_{j t}-S_{i t}\right) \cdot x_{j i t}^{k} \leq Q-S_{j t} \text {, } \\
& \forall i, j \in \mathcal{N}_{c}, k \in \mathcal{K}, t \in \mathcal{T} \\
& I_{t}=I_{t-1}+\sum_{k \in \mathcal{K}} \sum_{i, j \in \mathcal{N}, i \neq j} S_{i t} \cdot x_{i j t}^{k}-P_{t}, \quad \forall t \in \mathcal{T} \\
& P_{t} \leq \sum_{h \in \mathcal{H}} V_{h} \cdot \eta_{h t}, \quad \forall t \in \mathcal{T} \\
& y_{i t}^{k} \geq \sum_{j \in \mathcal{N}, i \neq j} S_{i t} \cdot x_{i j t}^{k}, \quad i \in \mathcal{N}, k \in \mathcal{K}, t \in \mathcal{T} \\
& y_{i t}^{k} \leq \sum_{j \in \mathcal{N}, i \neq j} Q \cdot x_{i j t}^{k}, \quad i \in \mathcal{N}, k \in \mathcal{K}, t \in \mathcal{T} \\
& I_{t} \leq V^{i n v}, \quad \forall t \in \mathcal{T} \\
& z_{t} \leq|\mathcal{K}|, \quad \forall t \in \mathcal{T} \\
& x_{i j t}^{k}, \eta_{l t} \in\{0,1\}, \quad \forall i, j \in \mathcal{N}, k \in \mathcal{K}, t \in \mathcal{T}, l \in \mathcal{L} \\
& z_{t} \in \mathbb{Z}^{+}, \forall t \in \mathcal{T} \\
& y_{i t}^{k}, I_{t}, P_{t} \geq 0, \forall l \in \mathcal{L}, i \in \mathcal{N}, k \in \mathcal{K}, t \in \mathcal{T}
\end{aligned}
$$

Objective (1) is to minimize the system cost, including the handling module operating cost, i.e., $\sum_{t \in \mathcal{T}} \sum_{h \in \mathcal{H}} C_{h}^{M} \eta_{h t}$, the vehicle deployment cost, i.e., $\sum_{t \in \mathcal{T}} C^{f} z_{t}$, the vehicle traveling cost, i.e., $\sum_{t \in \mathcal{T}} \sum_{k \in \mathcal{K}} \sum_{i, j \in \mathcal{N}, i \neq j} c_{i j} x_{i j t}^{k}$, the inventory holding cost, i.e., $\sum_{t \in \mathcal{T}} C^{i n v} I_{t}$, and the cost for disassembling EOL products, i.e., $\sum_{t \in \mathcal{T}} C^{d} P_{t}$. In objective function $(2), \operatorname{Prob}_{\mathbb{P}}(\cdot)$ is used to denote the probability of the event included in the parentheses under prob- 
ability distribution $\mathbb{P}$. Objective function (2) is to maximize the average customer service level.

Constraints (3) ensure that one collection center can be visited at most once by any vehicle during period $t$. Constraints (4) guarantee that each vehicle is deployed at most one time during period $t$. Constraints (5) define the number of vehicles deployed during each period. Constraints (6) serve as the flow conservation. Constraints (7) ensure that one handling module is selected during each period. Constraints (8) guarantee that the capacity of vehicle $k$ is respected at each node. Constraints (9) serve as the subtour elimination. Constraints (10) estimate the inventory level during each period. Constraints (11) respect the disassembly capacity under the selected handling module. Constraints (12) and (13) give the limitation of vehicle load. Constraints (14) are inventory capacity respect. Constraints (15) mean that the number of employed vehicles cannot exceed the total number of vehicles. Constraints (16)-(18) provides the domains of decision variables.

\section{Solution approaches}

As indicated by Birge and Louveaux (2011), it is relatively difficult to find exact solutions for general stochastic optimization problems. The studied problem is really complicated to be exactly solved, because the probability of uncertain parameters is partial known, and one of the objective functions is probabilistic. To better solve the problem, an equivalent transformation of the original objective function is firstly proposed, leading to an equivalent formulation [P2]. Then two common approximation methods, i.e., the SAA method and the ambiguity-set-based approximation method, are applied for the problem. The $\epsilon$-constraint method is further adapted to solve the problem.

\subsection{An equivalent formulation}

In this part, to tackle the probabilistic objective function (2), we first introduce new decision variables $\alpha$ and $\alpha_{l t}$, where $0 \leq \alpha, \alpha_{l t} \leq 1$, denot-

ing average customer service level and individual customer service level of demand of component $l \in \mathcal{L}$ during period $t \in \mathcal{T}$, respectively. Objective 
function (2) can be equivalently transformed into:

$$
\begin{aligned}
\max & \{\alpha\} \\
\text { s.t. } & \inf _{\mathbb{P}}\left\{\operatorname{Prob}_{\mathbb{P}}\left(n_{l} P_{t} \geq \xi_{l t}\right)\right\} \geq \alpha_{l t}, \quad \forall l \in \mathcal{L}, t \in \mathcal{T} \\
& \sum_{l \in \mathcal{L}} \sum_{t \in \mathcal{T}} \theta_{l t} \alpha_{l t}=\alpha \\
& 0 \leq \alpha_{l t}, \alpha \leq 1, \quad \forall l \in \mathcal{L}, t \in \mathcal{T}
\end{aligned}
$$

where $\inf _{\mathbb{P}}\{\cdot\}$ is used to imply the minimum value in the parentheses under probability distribution $\mathbb{P}$. Note that only partial knowledge of $\mathbb{P}$ is given. By introducing a new decision variable $\beta=1-\alpha$ and $0 \leq \beta \leq 1$, which is called the risk level, objective function (2) is equivalent to:

$\min \beta$

s.t. $(19)$

$$
\begin{aligned}
& \sum_{l \in \mathcal{L}} \sum_{t \in \mathcal{T}} \theta_{l t} \alpha_{l t}=1-\beta \\
& 0 \leq \alpha_{l t}, \beta \leq 1, \quad \forall l \in \mathcal{L}, t \in \mathcal{T}
\end{aligned}
$$

According, the original formulation $[\mathbf{P} \mathbf{1}]$ can be equivalently rewritten as the following bi-objective model $[\mathbf{P 2}]$ :

[P2] : $\min f_{1}$

$$
\begin{aligned}
& \min f_{2}^{\prime}=\beta \\
& \text { s.t. }(3)-(19),(22),(23)
\end{aligned}
$$

According to Sun et al. (2014), if $\alpha$ is a given parameter and $\theta_{l t}=1$, i.e., for equality $\sum_{l \in \mathcal{L}} \sum_{t \in \mathcal{T}} \alpha_{l t}=\alpha$, it is difficult to find the optimal combination of $\alpha_{l t}$. For our problem, $\alpha$ and $\alpha_{l t}$ are considered as a decision variable and $\sum_{l t} \theta_{l t}=1$ and $\sum_{l \in \mathcal{L}} \sum_{t \in \mathcal{T}} \theta_{l t} \alpha_{l t}=\alpha$, in the following, we consider $\alpha=\alpha_{l t}, \forall l \in \mathcal{L}, t \in \mathcal{T}$. Due to the partial known probability distribution $\mathbb{P}$ and the chance constraint (19), it is intractable to solve $[\mathbf{P 2}]$. Therefore, approximation methods are applied in the following.

\subsection{The $S A A$}

The SAA focuses on solving the stochastic optimization programs, by using Monte Carlo simulation (i.e., a finite set of scenarios) and techniques for 
deterministic optimization problems (Kleywegt et al., 2002). For the problem, only partial distributional information, i.e., the empirical mean and covariance of demands, is given. Based on the basic idea of the SAA, the partial known probability distribution $\mathbb{P}$ is replaced by an empirical one, corresponding to a finite set $\Omega$ of randomly generated scenarios which satisfy the given information (Nemirovski and Shapiro, 2006; Pagnoncelli et al., 2009). Following the idea in Zhang et al. (2015) and Jebali and Diabat (2017), we construct an SAA-based model [P3] with expected penalty, via introducing a new decision variable $\zeta_{l t}(\omega)$ to estimate the unmet demands of component $l$ at period $t$ under scenario $\omega \in \Omega$. In the model, the minimization of $f_{2}^{\prime}=\beta$ and chance constraint (19) are approximated by minimizing the expected sum of penalty for $\zeta_{l t}(\omega)$. Before constructing the model [P3], additional parameters and decision variables are presented as follows:

\section{New parameters:}

- $\Omega$ : Set of scenarios indexed by $\omega$, and $\Omega=\{1,2, \ldots,|\Omega|\}$.

- $\xi_{l t}(\omega)$ : Demand of component $l \in \mathcal{L}$ in period $t \in T$ under scenario $\omega \in \Omega$.

- CP: Unit penalty cost for unmet demand.

\section{New decision variables:}

- $\zeta_{l t}(\omega)$ : Continuous variable, unmet demand of component $l \in \mathcal{L}$ during period $t$ under scenario $\omega \in \Omega$.

\section{[P3] :}

$$
\begin{aligned}
\min f_{1} & =\left\{\sum_{t \in \mathcal{T}}\left(\sum_{h \in \mathcal{H}} C_{h}^{M} \eta_{h t}+C^{f} z_{t}+\sum_{k \in \mathcal{K}} \sum_{i, j \in \mathcal{N}, i \neq j} c_{i j} x_{i j t}^{k}+C^{i n v} I_{t}+C^{d} P_{t}\right)\right\} \\
\min f_{2}^{\prime \prime} & =\frac{C P}{|\Omega|} \sum_{\omega \in \Omega} \sum_{t \in \mathcal{T}} \sum_{l \in \mathcal{L}} \zeta_{l t}(\omega)
\end{aligned}
$$

s.t. $(3)-(17)$

$$
\begin{aligned}
& n_{l} P_{t}+\zeta_{l t}(\omega) \geq \xi_{l t}(\omega), \quad \forall l \in \mathcal{L}, t \in \mathcal{T}, \omega \in \Omega \\
& \zeta_{l t}(\omega) \geq 0, \quad \forall l \in \mathcal{L}, t \in \mathcal{T}, \omega \in \Omega
\end{aligned}
$$

The objective function $f_{2}^{\prime \prime}$ in (24) focuses on minimizing the expected sum of penalty cost for unmet demand $\zeta_{l t}(\omega)$. The $\epsilon$-constraint method for 
multi-optimization ean be applied to solve the mixed integer programming formulation [P3] in Section 4.4. We observe that the computational time of the SAA increases dramatically with the problem size, due to the NP-hard nature of the problem. Thus we adopt another approximation method in the following, to achieve computational efficiency.

\subsection{The approximated MIP model}

In this part, we first construct an ambiguity set, which is based on the known empirical mean and covariance matrix of demands, to portray the partial known distribution $\mathbb{P}$. Then a set of individual chance constraints is then applied to conservatively approximate the chance constraint (19), to construct an approximated MIP model $[\mathbf{P} 4]$.

\subsubsection{The ambiguity set}

For the partial known distributional information, a common method is to construct an ambiguity set, which is assumed to include a family of probability distributions that satisfy the given information. The ambiguity set introduced by Delage and Ye (2010), which takes the inevitable estimation errors into consideration, has been widely and successfully used (Cheng et al., 2013; Zhang et al., 2017). In the work, following Delage and Ye (2010), we introduce an ambiguity set $\mathcal{P}\left(\boldsymbol{\mu}, \boldsymbol{\Gamma}, \gamma_{1}, \gamma_{2}\right)$ for the uncertain-vector $\boldsymbol{\xi}$ :

$$
\mathcal{P}\left(\boldsymbol{\mu}, \boldsymbol{\Gamma}, \gamma_{1}, \gamma_{2}\right)=\left\{\begin{array}{ll}
P: & \left(\mathbb{E}_{P}[\boldsymbol{\xi}]-\boldsymbol{\mu}\right)^{\top}(\boldsymbol{\Sigma})^{-1}\left(\mathbb{E}_{P}[\boldsymbol{\xi}]-\boldsymbol{\mu}\right) \leq \gamma_{1}, \\
& \mathbb{E}_{P}\left[(\boldsymbol{\xi}-\boldsymbol{\mu})(\boldsymbol{\xi}-\boldsymbol{\mu})^{\top}\right] \preceq \gamma_{2} \boldsymbol{\Gamma} .
\end{array}\right\},
$$

where $\mathbb{E}[\cdot]$ implies the expected value, and $P$ denotes the candidate probability distribution, and $\mathcal{P}$ is an ambiguity set in which the partial known distribution $\mathbb{P}$ is assumed to be included in it, and $\gamma_{1} \geq 0$ and $\gamma_{2} \geq \gamma_{1}$ are two parameters which restrict that: (i) the true mean vector of $\boldsymbol{\xi}$ is in an ellipsoid with center $\boldsymbol{\mu}$ and radius $\gamma_{1}$; (ii) the true covariance matrix of $\boldsymbol{\xi}$ is in a positive semi-definite cone bounded by $\gamma_{2} \boldsymbol{\Gamma}$. Accordingly, chance constraint (19) can be approximated as:

$$
\begin{aligned}
& \inf _{\mathbb{P} \in \mathcal{P}}\left\{\operatorname{Prob}_{\mathbb{P}}\left(n_{l} P_{t} \geq \xi_{l t}\right)\right\} \geq \alpha_{l t}, \quad \forall l \in \mathcal{L}, t \in \mathcal{T} \\
\Leftrightarrow & \inf _{\mathbb{P} \in \mathcal{P}}\left\{\operatorname{Prob}_{\mathbb{P}}\left(\xi_{l t} \leq n_{l} P_{t}\right)\right\} \geq \alpha_{l t}, \quad \forall l \in \mathcal{L}, t \in \mathcal{T}
\end{aligned}
$$




\subsubsection{The approximated model}

By introducing a vector $\boldsymbol{\pi}_{l t}=[0, \ldots, 0,1,0, \ldots, 0]^{\top}$, where the $((l-1) \cdot|\mathcal{T}|+$ $t$ )-th element is equal to 1 , and other elements are 0 , item $\xi_{l t}$ in Constraint (27) can be rewritten as $\boldsymbol{\pi}_{l t}^{\top} \boldsymbol{\xi}$, thus Constraint (27) is equivalent to:

$$
\inf _{\mathbb{P} \in \mathcal{P}}\left\{\operatorname{Prob}_{\mathbb{P}}\left(\boldsymbol{\pi}_{l t}^{\top} \boldsymbol{\xi} \leq n_{l} P_{t}\right)\right\} \geq \alpha_{l t}, \quad \forall l \in \mathcal{L}, t \in \mathcal{T}
$$

Constraint (28) can be approximated based on the method introduced by Zhang et al. (2017) as:

$$
\begin{gathered}
\sqrt{\frac{1}{1-a-b}}\left(1+\sqrt{\frac{\left(1-\alpha_{l t}\right) b}{\alpha_{l t}}}\right) \sqrt{\boldsymbol{\pi}_{l t}^{\top} \boldsymbol{\Sigma} \boldsymbol{\pi}_{l t}} \leq \quad \sqrt{\frac{1-\alpha_{l t}}{\alpha_{l t}}}\left(n_{l} P_{t}-\boldsymbol{\mu}^{\top} \boldsymbol{\pi}_{l t}\right), \\
\forall l \in \mathcal{L}, t \in \mathcal{T}
\end{gathered}
$$

where parameters $a, b$ and $\gamma_{1}, \gamma_{2}$ satisfy the following equalities:

$\gamma_{1}=\frac{b}{1-a-b}, \quad \gamma_{2}=\frac{1+b}{1-a-b}$

Then an approximated bi-objective MIP model $[\mathbf{P 4}]$ can be formulated:

$[\mathbf{P} 4]: \min f_{1}$

$$
\min f_{2}^{\prime \prime \prime}=\beta
$$$$
\text { s.t. }(3)-(18),(22),(23),(29)
$$

\subsection{The $\epsilon$-constraint framework}

To solve the above approximated bi-objective formulations $[\mathbf{P} 3]$ and $[\mathbf{P 4}]$, we apply a classic $\epsilon$-constraint method, to solve them.

For the sake of simplicity, the bi-objective optimization problem can be rewritten as: $\min \left\{f_{1}(\boldsymbol{x}), f_{2}(\boldsymbol{x}) \mid \boldsymbol{x} \in \mathcal{X}\right\}$, where $f_{1}(\boldsymbol{x})$ and $f_{2}(\boldsymbol{x})$ denote the above system cost $f_{1}$ and the risk level $f_{2}^{\prime}=\beta$ in [P2], and $\boldsymbol{x}$ denotes a decision variable vector with feasible region $\mathcal{X}$ defined by Constraints (3)(19), (22) and (23). A non-dominated solution can be defined as follows: a solution $\boldsymbol{x}$ is non-dominated if there exists no solution $\boldsymbol{x}^{\prime} \in \mathcal{X}$ with $f_{1}\left(\boldsymbol{x}^{\prime}\right) \leq$ $f_{1}(\boldsymbol{x})$ and $f_{2}\left(\boldsymbol{x}^{\prime}\right)<f_{2}(\boldsymbol{x})$ or $f_{1}\left(\boldsymbol{x}^{\prime}\right)<f_{1}(\boldsymbol{x})$ and $f_{2}\left(\boldsymbol{x}^{\prime}\right) \leq f_{2}(\boldsymbol{x})$ (T'kindt and Billaut, 2001). The two objective values of a non-dominated solution 
correspond to a non-dominated point, and all non-dominated points form the Pareto front.

The basic idea of the $\epsilon$-constraint method is to iteratively optimize transformed single-objective $\epsilon$-constraint problems, in which the main objective is considered as their objectives and another one is restricted by $\epsilon$ and added as $\epsilon$ constraints:

$\min f_{1}(\boldsymbol{x})$

s.t. $f_{2}(\boldsymbol{x}) \leq \epsilon, \quad \forall \boldsymbol{x} \in \mathcal{X}$

The definition of the value of $\epsilon$ during each iteration is critical, and the range of $\epsilon$ is denoted by $\left[f_{2}^{I}, f_{2}^{N}\right]$, determined by solving the following singleobjective problems (Bérubé et al., 2009): (i) $f_{1}^{I}=\min _{\boldsymbol{x} \in \mathcal{X}}\left\{f_{1}(\boldsymbol{x})\right\}$ and $f_{2}^{I}=$ $\min _{\boldsymbol{x} \in \mathcal{X}}\left\{f_{2}(\boldsymbol{x})\right\} ;$ (ii) $f_{1}^{N}=\min _{\boldsymbol{x} \in \mathcal{X}}\left\{f_{1}(\boldsymbol{x}): f_{2}(\boldsymbol{x})=f_{2}^{I}\right\}$ and $f_{2}^{N}=\min _{\boldsymbol{x} \in \mathcal{X}}\left\{f_{2}(\boldsymbol{x})\right.$ : $\left.f_{1}(\boldsymbol{x})=f_{1}^{I}\right\}$. The framework of the $\epsilon$-constraint method is presented in Algorithm 1.

For formulation $[\mathbf{P 3}]$, we first calculate $f_{1}^{I}, f_{2}^{\prime \prime I}, f_{1}^{N}$ and $f_{2}^{\prime \prime N}$. At each iteration, the first objective $f_{1}$ is minimize by adding constraint $f_{2}^{\prime \prime} \leq \epsilon_{1}$, to form $[\mathbf{P 3}(\epsilon)]$, with $\epsilon \in\left[f_{2}^{\prime \prime I}, f_{2}^{\prime \prime N}\right]$. For formulation $[\mathbf{P 4}]$, we also minimize the first objective function $f_{1}$ by adding constraint $f_{2}^{\prime \prime \prime} \leq \epsilon_{2}$, i.e., $\beta \leq \epsilon_{2}$, to form $[\mathbf{P} 4(\epsilon)]$ during each iteration. As $0 \leq \beta \leq 1$, the range of $\epsilon_{2}$ is $[0,1]$. Note that the step sizes of $\epsilon_{1}$ and $\epsilon_{2}$ are denoted by $\Delta_{1}$ and $\Delta_{2}$, respectively.

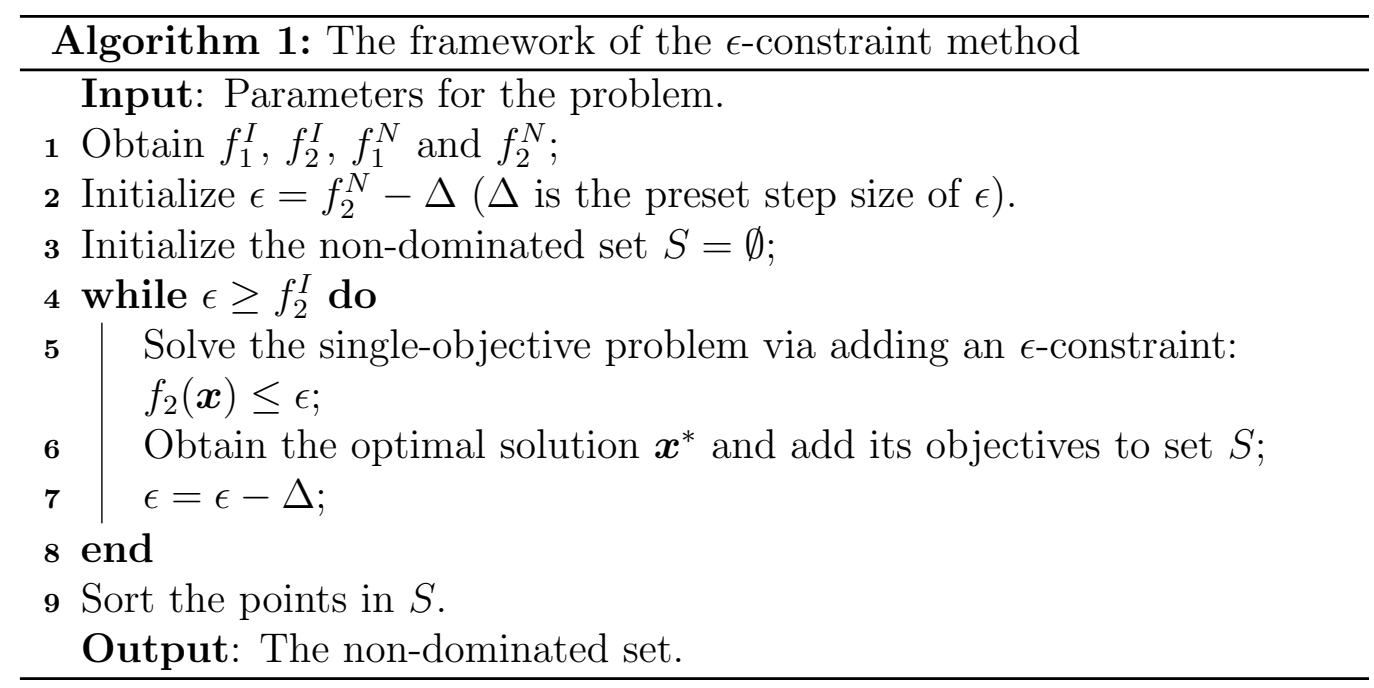




\section{Computational experiments}

In this section, the proposed two approximated models are solved by the $\epsilon$-constraint method. The transformed single-objective $\epsilon$-constrained models $[\mathbf{P 3}(\epsilon)]$ and $[\mathbf{P 4}(\epsilon)]$ are coded in MATLAB_2014b and solved by calling CPLEX12.6 solver. All numerical experiments are conducted on a personal computer with Core I7 and 3.60GHz processor and 8GB RAM under Windows 7 Operation System. Each instance is tested 10 times to obtain its average value.

\subsection{Out-of-sample test}

We the nominated solutions-obtained by solving the two formations $[\mathbf{P 3}]$ and $[\mathbf{P 4}]$, via the $\epsilon$-constraint method, in a large set of randomly generated scenarios, i.e., the out-of-sample test (Zhang et al., 2017; Zhang et al., 2018; Liu et al., 2019). In each scenario, demands of components are randomly generated following a Log-Normal distribution, satisfying the known mean vector $\boldsymbol{\mu}$ and covariance matrix $\boldsymbol{\Gamma}$ (Xie and Ahmed, 2018). The number of tested scenarios is set to be 10000 in this work. For each nondominated solution obtained by the $\epsilon$-constraint method via solving each formulation, based on its obtained vehicle deployment, vehicle routes, handling module selection and the number of disassembled EOL products and inventory level during each period,

(1) Under each scenario $\omega$ out of the 10000 ones, the (out-of-sample) individual service level $\alpha_{l t}(\omega)$, i.e., $\alpha_{l t}(\omega)=1$ if $n_{l} P_{t} \geq \xi_{l t}(\omega)$, and $\alpha_{l t}(\omega)=\frac{n_{l} P_{t}}{\xi_{l t}(\omega)}$ otherwise, can be easily calculated.

(2) Then the (out-of-sample) average risk level (i.e., the second objective $f_{2}^{\prime}=\beta$ in $\left.[\mathbf{P 2}]\right)$, which is estimated by $\left(1-\sum_{l \in \mathcal{L}} \sum_{t \in \mathcal{T}} \theta_{l t} \frac{\sum_{\omega=1}^{10000} \alpha_{l t}(\omega)}{10000}\right) \times$ $100 \%$, can be calculated.

The following well-known metrics are used to evaluate the out-of-sample performance:

(i) The number of non-dominated solutions.

(ii) Inverted generational distance (Coello and Cortés, 2005): 
$I_{D}\left(A, P^{*}\right)=\frac{1}{P^{*}} \sum_{x^{\prime} \in P^{*}} \min _{\boldsymbol{x} \in A}\left\{d\left(\boldsymbol{x}, \boldsymbol{x}^{\prime}\right)\right\}$

where $A$ denotes the obtained set of non-dominated solutions, and $P^{*}$ is the reference set, and $\boldsymbol{x}$ and $\boldsymbol{x}^{\prime}$ denote the solutions. As the optimal Pareto solutions cannot be obtained for the problem, we consider that $P^{*}$ includes all non-dominated solutions obtained by the two formulations via $\epsilon$-constraint method, as in line with Liu et al. (2018). Besides, $d\left(\boldsymbol{x}, \boldsymbol{x}^{\prime}\right)$ denotes the Euclidean distance between solutions $\boldsymbol{x}$ and $\boldsymbol{x}^{\prime}$, which is calculated as

$d\left(\boldsymbol{x}, \boldsymbol{x}^{\prime}\right)=\sqrt{\left(\frac{f_{1}(\boldsymbol{x})-f_{1}\left(\boldsymbol{x}^{\prime}\right)}{f_{1}^{\max }-f_{1}^{\min }}\right)^{2}+\left(\frac{f_{2}(\boldsymbol{x})-f_{2}\left(\boldsymbol{x}^{\prime}\right)}{f_{2}^{\max }-f_{2}^{\min }}\right)^{2}}$

where $f_{1}^{\max }$ and $f_{1}^{\min }$ denote the maximum and minimum system costs (i.e., the first objective in [P2]) of all non-dominated solutions in the reference set $P^{*}$, and $f_{2}^{\max }$ and $f_{2}^{\min }$ are the maximum and minimum (out-of-sample) risk levels (i.e., the second objective in [P2]) of all non-dominated solutions in the reference set $P^{*}$. A smaller $I_{D}\left(A, P^{*}\right)$ implies that the non-dominated set $A$ approximates the reference set $P^{*}$ better.

(iv) Set coverage indicator (Zitzler et al., 2000):

$C(A, B)=\frac{|\boldsymbol{x} \in B| \exists \boldsymbol{x}^{\prime} \in A: \boldsymbol{x}^{\prime} \succeq \boldsymbol{x} \mid}{|B|}$,

where $A$ and $B$ are the obtained non-dominated sets obtained by the two formulations [P3] and [P4] via $\epsilon$-constraint method, and $\boldsymbol{x} \in A$ and $\boldsymbol{x}^{\prime} \in B$, and $\boldsymbol{x}^{\prime} \succeq \boldsymbol{x}$ denotes that solution $\boldsymbol{x}^{\prime}$ dominates solution $\boldsymbol{x}$. It can be obtained that $C(A, B)=1$ if all solutions in $B$ are dominated by some solutions in $A$. That is, if $C(A, B) \geq C(B, A), A$ is better than $B$ in terms of dominance relation. 
(v) Maximum spread indicator (Liu et al., 2016; Liu et al., 2018):

$$
M S(A)=\sqrt{\sum_{i=1}^{2}\left(\max _{\boldsymbol{x} \in A} \frac{f_{i}(\boldsymbol{x})-f_{i}^{\text {min }}}{f_{i}^{\text {max }}-f_{i}^{\text {min }}}-\min _{\boldsymbol{x} \in A} \frac{f_{i}(\boldsymbol{x})-f_{i}^{\text {min }}}{f_{i}^{\text {max }}-f_{i}^{\text {min }}}\right)^{2}}
$$

$M S(A)$ evaluates the distance between the boundary solutions in a set $A$ of non-dominated solutions. A larger $M S(A)$ denotes a wider range of objective values covered by set $A$.

Besides, we also use the average standard deviation $\sigma=\sum_{l \in \mathcal{L}} \sum_{t \in \mathcal{T}} \theta_{l t} \sigma_{l t}$, where $\sigma_{l t}$ denotes the standard deviation of the individual service level $\alpha_{l t}$, i.e., $\sigma_{l t}=\frac{1}{10000} \sum_{\omega=1}^{10000}\left(\alpha_{l t}(\omega)-\mathbb{E}\left(\alpha_{l t}\right)\right)^{2}$ and $\mathbb{E}\left(\alpha_{l t}\right)=\frac{1}{10000} \alpha_{l t}(\omega)$. Note that a smaller $\sigma$ implies that the solutions are more stable, in terms of the service level.

\subsection{An illustrative example}

Preliminary experiments are analysed, to adjust the input parameters. Parameters are shown in Table 1, where (i) for the SAA model [P3], the number $|\Omega|$ of scenarios and the unit penalty $\operatorname{cost} \theta_{1}$ for unmet demand are set to be 200 and 1 , and the step size $\Delta$ and 1 (i.e., the smallest unit of penalty cost), respectively; and (ii) for the approximated MIP model [P4], $\gamma_{1}$ and $\gamma_{2}$ are set to be 0.8 and 1 , and the step size $\Delta_{2}$ is set to be 0.05 for efficiency. Besides, without loss of generality, $\theta_{l t}$ is set to be $\frac{1}{|\mathcal{L}| \cdot|\mathcal{T}|}$.

Table 1: Parameters for the solution methods

\begin{tabular}{ccccccccc}
\hline & \multicolumn{3}{c}{ SAA model [P3] } & & \multicolumn{3}{c}{ Approximated MIP model [P4] } \\
\cline { 2 - 5 } \cline { 6 - 8 } Parameters & $|\Omega|$ & $C P$ & $\Delta_{1}$ & & $\gamma_{1}$ & $\gamma_{2}$ & $\Delta_{2}$ \\
Values & 200 & 1 & 1 & & 0.8 & 1 & 0.05 \\
\hline
\end{tabular}

An illustrative example based on a small network is investigated, to compare the proposed two approximated models. The parameters of the example are detailed in Table 2, where there are 4 collection centers, 10 time periods, 5 components, 5 vehicles and 3 alternative handling modules in total. The data is generated as in line with Habibi et al. (2017a), which focus on the deterministic and single-objective collection-disassembly problem, such that (i) the inventory capacity $V^{i n v}$ is set to be 50; (ii) the fixed vehicle deploying 
cost $C^{f}$, the traveling $\operatorname{cost} c_{i j}$ from node $i$ to $j$, the unit inventory cost $C^{i n v}$ and the unit disassembly cost $C^{d}$ are set to be 10, 1, 1 and 10, respectively; (iii) the vehicle capacity is set to be $2 \cdot \frac{\sum_{i \in \mathcal{N}_{c}} S_{i t}}{|\mathcal{T}|}$; (iv) $S_{i t}, \mu_{l t}$ are randomly and uniformly generated from intervals $[9,11]$ and $[0.6,1.4] \cdot \frac{\sum_{i \in \mathcal{N}_{c}} \sum_{t \in \mathcal{T}} S_{i t}}{|\mathcal{N}| \cdot|\mathcal{T}|}$. The fixed operating costs and disassembly line capacities for the 3 handling modules are $[10,20,30]$ and $[10,20,30]$. The correlation between demands depends on the specific application. To illustrate the proposed approximated models, we assume there is a neutral correlation, i.e., demands are assumed to be independently distributed (Chen et al., 2010). The standard deviation of each demand $\xi_{l t}$ is set to be $0.2 \cdot \mu_{l t}$.

Table 2: Input parameters for the small network

\begin{tabular}{cccccccccccc}
\hline & Collection centers $\backslash$ Periods & 1 & 2 & 3 & 4 & 5 & 6 & 7 & 8 & 9 & 10 \\
\cline { 2 - 10 }$S_{i t}$ & 1 & 11 & 11 & 10 & 9 & 11 & 10 & 10 & 10 & 9 & 11 \\
& 2 & 11 & 9 & 9 & 9 & 9 & 10 & 9 & 10 & 9 & 9 \\
& 3 & 10 & 11 & 10 & 9 & 10 & 9 & 11 & 11 & 9 & 9 \\
& 4 & 10 & 11 & 10 & 11 & 9 & 11 & 11 & 11 & 10 & 11 \\
& Components $\backslash$ Periods & 1 & 2 & 3 & 4 & 5 & 6 & 7 & 8 & 9 & 10 \\
\cline { 2 - 10 } & 1 & 11 & 5 & 9 & 11 & 8 & 6 & 11 & 7 & 5 & 8 \\
& 2 & 11 & 10 & 9 & 10 & 9 & 11 & 11 & 10 & 11 & 11 \\
& 3 & 8 & 10 & 6 & 5 & 5 & 11 & 5 & 5 & 6 & 6 \\
& 4 & 8 & 9 & 5 & 8 & 11 & 9 & 5 & 6 & 8 & 11 \\
& 5 & 10 & 7 & 6 & 6 & 8 & 7 & 6 & 10 & 6 & 8 \\
\hline
\end{tabular}

Pareto fronts obtained by the SAA method and the approximated MIP model are illustrated in Figure 2. Comparison between the two methods for the illustrative example is reported in Table 3, where "Num" denotes the number of non-dominated solutions. We can observe from Table 3 that the computational time of the SAA is 869.4 seconds, which is about 8.3 times larger than that of the approximated MIP model. Besides, it can also be observed from Figure 2 and Table 3 that the SAA method can obtain a larger number of non-dominated solutions than the approximated MIP model. Besides, the inverted generational distances of the SAA method is 0.062, which is slightly smaller than that of the approximated MIP model. That is, the SAA method performs better, in terms of the number of nondominated solutions and the $I_{D}$ indicator. However, the average standard deviation $\sigma$ of the service level of the non-dominant set obtained by the SAA 


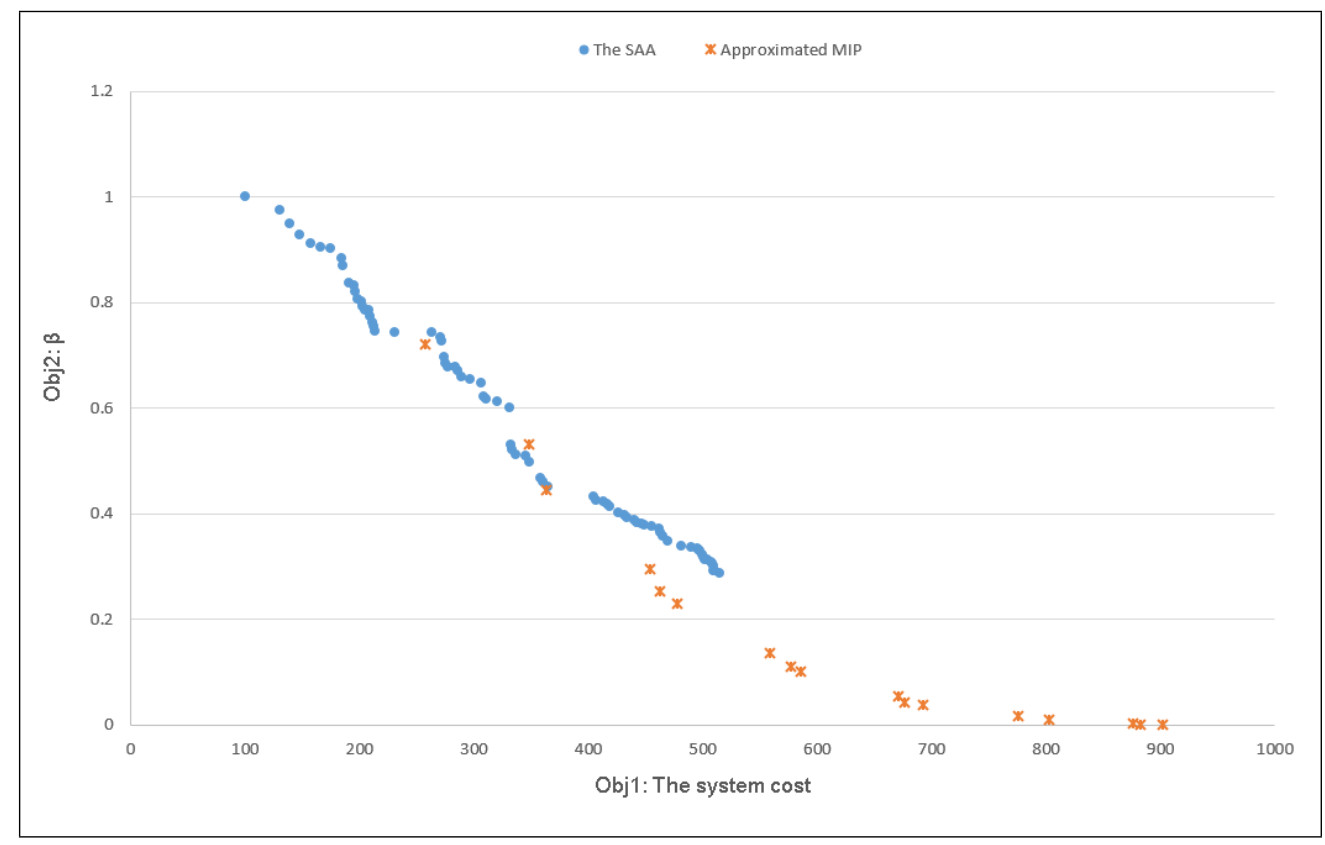

Figure 2: Pareto fronts obtained by the two solution methods for the illustrative example

is 0.060, which is a little larger than that of the approximated MIP model. The $C(B, A)$ of the approximated MIP model is 0.312 , and $C(A, B)$ of the SAA method is 0.062 . In terms of the maximum spread indicator MS, the approximated MIP model can obtain non-dominated solutions covering a wider range of objective values. Moreover, we can also observe from Figure 2 that the approximated MIP can obtain solutions with larger service levels, and the SAA can obtain solutions with smaller costs.

In sum, the numerical results of the tested instance show that the SAA can obtains more non-dominated solutions. The approximated MIP performs better, in terms of the dominance relation, the range of objective values and the computational time.

Table 3: Comparison between the two methods for the illustrative example

\begin{tabular}{|c|c|c|c|c|c|c|c|c|c|c|c|}
\hline \multicolumn{6}{|c|}{ The SAA (referred to as A) } & \multicolumn{6}{|c|}{ Approximated MIP model (referred to as B) } \\
\hline Time $(\mathrm{s})$ & $\sigma$ & Num & $I_{D}$ & $C(A, B)$ & $M S$ & Time $(\mathrm{s})$ & $\sigma$ & Num & $I_{D}$ & $C(B, A)$ & $M S$ \\
\hline 869.4 & 0.060 & 77 & 0.062 & 0.058 & 0.881 & 93.4 & 0.056 & 17 & 0.078 & 0.312 & 1.075 \\
\hline
\end{tabular}




\subsection{Computational results}

Computational experiments on randomly generated instances of different scales are conducted. The parameters are set as mentioned above. Besides, the problem scales of the tested instances are in line with Habibi et al. (2019). Numerical results are reported in Table 4 , where the first 4 columns report the problem scales, including the number of nodes $|\mathcal{N}|$, the number of periods $|\mathcal{T}|$, the number of components $|\mathcal{A}|$ and the number of the vehicles $|\mathcal{K}|$, respectively.

In Table 4, we mark the better metrics in average in bold. The average computational time of the approximated MIP model is 130.1 seconds, which is only $6.55 \%$ of that obtained by the SAA model. In terms of the average standard deviation $\sigma$ of the customer service level, the approximated MIP performs better, and is about $36.95 \%$ smaller than that of the SAA method. That means, the non-dominated solutions obtained by the approximated MIP model are more stable, in terms of the customer service level. In terms of set coverage indicator $C(A, B)$, we can observe that obtained by the approximated MIP model is 0.308, which is larger than that of the SAA method. That is, the approximated MIP model performs better in terms of dominance relation. We can also observe that the number of non-dominated solutions and the average inverted generational distance obtained by the SAA method are better than those proposed by the approximated MIP model. Besides, the SAA method achieves larger values, in terms of $M S$ indicator.

According to the above numerical results, we obtain that the SAA method performs better than the approximated MIP model, in terms of the number, the efficiency and the range of non-dominated solutions. The approximated MIP model has very great superiorities, in terms of the computational time, the stability of customer service level and the dominance relations of its non-dominated solutions. In sum, the SAA model may be chosen, if a large number of solutions are required and the decision maker can wait for a long time. Otherwise, we recommend the approximated MIP model, as it can obtain solutions with high quality within a reasonable time.

\section{Conclusion}

This work investigate a stochastic collection-disassembly problem with handling module selection for disassembly site, to minimize the system cost and maximize the average customer service level. Customer demands of components that can be reused are assumed to be stochastic, and only partial 


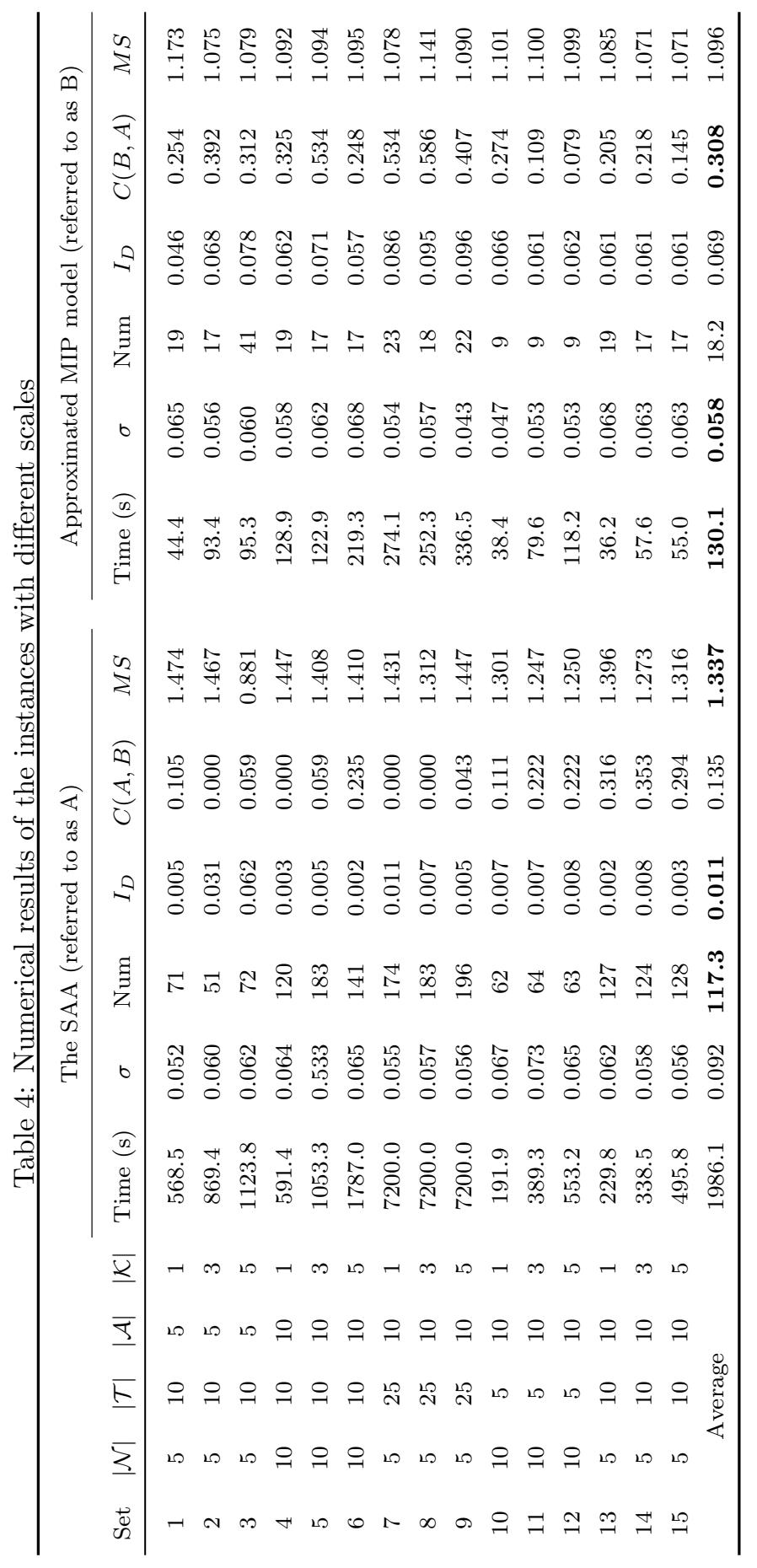


information on the probability distribution is known (i.e., the empirical mean vector and covariance matrix). For the problem, we first construct a distributionally robust bi-objective formulation. The formulation cannot be directly solved by calling off-the-shelf solvers, thus we equivalently transform the formulation into a distributionally robust bi-objective chance constrained model. An SAA-based model and an approximated MIP model are constructed and solved via the $\epsilon$-constraint method framework. Various numerical experiments are conducted, to evaluate the applicability and the performance of the two models. Numerical results show that the approximated MIP model can obtain solutions with high quality within a reasonable time.

Future research directions may include: (i) to develop bi-objective algorithms that can solve the problem more efficiently, (ii) to consider the uncertain amount of available EOL products at each collection center, and (iii) to take the environmental impacts of the remanufacturing process in to consideration.

\section{Acknowledgements}

This work was supported by the National Natural Science Foundation of China (NSFC) under Grants 71531011, 71771048 and 71571134. This work was also supported by the Fundamental Research Funds for the Central Universities.

\section{References}

[1] Agrawal, S. and Tiwari, M. K. (2008). A collaborative ant colony algorithm to stochastic mixed-model u-shaped disassembly line balancing and sequencing problem. International Journal of Production Research, 46(6):1405-1429.

[2] Bentaha, M. L., Battaïa, O., and Dolgui, A. (2012). A stochastic formulation of the disassembly line balancing problem. In IFIP International Conference on Advances in Production Management Systems, pages 397404. Springer, Berlin, Heidelberg.

[3] Bentaha, M. L., Battaïa, O., and Dolgui, A. (2013). L-shaped algorith$\mathrm{m}$ for stochastic disassembly line balancing problem. IFAC Proceedings Volumes, 46(9):407-411. 
[4] Bentaha, M. L., Battaïa, O., and Dolgui, A. (2014a). A sample average approximation method for disassembly line balancing problem under uncertainty. Computers $\&$ Operations Research, 51(3):111-122.

[5] Bentaha, M. L., Battaïa, O., and Dolgui, A. (2015). An exact solution approach for disassembly line balancing problem under uncertainty of the task processing times. International Journal of Production Research, 53(6):1807-1818.

[6] Bentaha, M. L., Battaïa, O., Dolgui, A., and Hu, S. J. (2014b). Dealing with uncertainty in disassembly line design. CIRP Annals - Manufacturing Technology, 63(1):21-24.

[7] Bérubé, J. F., Gendreau, M., and Potvin, J.-Y. (2009). An exact constraint method for bi-objective combinatorial optimization problems: Application to the traveling salesman problem with profits. European Journal of Operational Research, 194(1):39-50.

[8] Bijvank, M. and Vis, I. F. A. (2012). Lost-sales inventory systems with a service level criterion. European Journal of Operational Research, $220(3): 610-618$.

[9] Birge, J. R. and Louveaux, F. (2011). Introduction to Stochastic Programming. Springer Science \& Business Media, New York.

[10] Cardona-Valds, Y., lvarez, A., and Ozdemir, D. (2011). A bi-objective supply chain design problem with uncertainty. Transportation Research Part C, 19(5):821-832.

[11] Cardona-Valds, Y., lvarez, A., and Pacheco, J. (2014). Metaheuristic procedure for a bi-objective supply chain design problem with uncertainty. Transportation Research Part B, 60(5):66-84.

[12] Chen, W., Sim, M., Sun, J., and Teo, C. P. (2010). From cvar to uncertainty set: Implications in joint chance-constrained optimization. Operations Research, 58(2):470-485.

[13] Cheng, J., Lisser, A., and Letournel, M. (2013). Distributionally robust stochastic shortest path problem. Electronic Notes in Discrete Mathematics, 41(41):511-518. 
[14] Coello, C. A. C. and Cortés, N. C. (2005). Solving multiobjective optimization problems using an artificial immune system. Genetic Programming and Evolvable Machines, 6(2):163-190.

[15] Delage, E. and Ye, Y. (2010). Distributionally robust optimization under moment uncertainty with application to data-driven problems. Operations Research, 58(3):595-612.

[16] Habibi, M. K., Battaïa, O., Cung, V.-D., and Dolgui, A. (2017a). Collection-disassembly problem in reverse supply chain. International Journal of Production Economics, 183:334-344.

[17] Habibi, M. K. K., Battaïa, O., Cung, V. D., and Dolgui, A. (2017b). An efficient two-phase iterative heuristic for collection-disassembly problem. Computers $\mathcal{E}$ Industrial Engineering, 110:505-514.

[18] Habibi, M. K. K., Battaïa, O., Cung, V.-D., Dolgui, A., and Tiwari, M. K. (2019). Sample average approximation for multi-vehicle collectiondisassembly problem under uncertainty. International Journal of Production Research, 57(8):2409-2428.

[19] Ilgin, M. A. and Gupta, S. M. (2010a). Comparison of economic benefits of sensor embedded products and conventional products in a multi-product disassembly line. Computers \& Industrial Engineering, 59(4):748-763.

[20] Ilgin, M. A. and Gupta, S. M. (2010b). Environmentally conscious manufacturing and product recovery (ecmpro): A review of the state of the art. Journal of Environmental Management, 91(3):563-591.

[21] Ivanov, D., Pavlov, A., Pavlov, D., and Sokolov, B. (2017). Minimization of disruption-related return flows in the supply chain. International Journal of Production Economics, 183:503-513.

[22] Jebali, A. and Diabat, A. (2017). A chance-constrained operating room planning with elective and emergency cases under downstream capacity constraints. Computers $\mathcal{E} 3$ Industrial Engineering, 114:329-344.

[23] Kleywegt, A. J., Nori, V. S., and Savelsbergh, M. W. P. (2002). The stochastic inventory routing problem with direct deliveries. Transportation Science, 36(1):94-118. 
[24] Li, J., Ren, C., Dong, J., and He, M. (2011). A lagrangean-based heuristic for a two-stage facility location problem with handling costs. In IEEE International Conference on Service Operations, Logistics, and Informatics, pages 319-324.

[25] Liu, M., Lee, C.-Y., Zhang, Z., and Chu, C. (2016). Bi-objective optimization for the container terminal integrated planning. Transportation Research Part B, 93:720-749.

[26] Liu, M., Liu, X., Chu, F., Zheng, F., and Chu, C. (2019). Distributionally robust inventory routing problem to maximize the service level under limited budget. Transportation Research Part E, 126:190-211.

[27] Liu, M., Yang, X., Chu, F., Zhang, J., and Chu, C. (2018). Energyoriented bi-objective optimization for the tempered glass scheduling. Omega, pages 173-195.

[28] Mcgovern, S. M. and Gupta, S. M. (2007). A balancing method and genetic algorithm for disassembly line balancing. European Journal of Operational Research, 179(3):692-708.

[29] Nemirovski, A. and Shapiro, A. (2006). Convex approximations of chance constrained programs. SIAM Journal on Optimization, 17(4):969996.

[30] Özceylan, E., Kalayci, C. B., Güngör, A., and Gupta, S. M. (2018). Disassembly line balancing problem: a review of the state of the art and future directions. Internatioal Journal of Production Research. DOI:10.1080/00207543.2018.1428775.

[31] Özceylan, E. and Paksoy, T. (2014). Fuzzy mathematical programming approaches for reverse supply chain optimization with disassembly line balancing problem. Journal of Intelligent \&3 Fuzzy Systems, 26(4):19691985.

[32] Pagnoncelli, B. K., Ahmed, S., and Shapiro, A. (2009). Sample average approximation method for chance constrained programming: Theory and applications. Journal of Optimization Theory \& Applications, 142(2):399416 . 
[33] Paksoy, T. (2013). Mixed model disassembly line balancing problem with fuzzy goals. International Journal of Production Research, 51(20):60826096.

[34] Sun, H., Gao, Z., Szeto, W. Y., Long, J., and Zhao, F. (2014). A distributionally robust joint chance constrained optimization model for the dynamic network design problem under demand uncertainty. Networks 83 Spatial Economics, 14(3-4):409-433.

[35] Tiwari, M., K., Raghavendra, Agrawal, Shubham, Goyal, and S., K. (2010). A hybrid taguchi-immune approach to optimize an integrated supply chain design problem with multiple shipping. European Journal of Operational Research, 203(1):95-106.

[36] T'kindt, V. and Billaut, J.-C. (2001). Multicriteria scheduling problems: a survey. RAIRO-Operations Research, 35(2):143-163.

[37] Tuncel, E., Zeid, A., and Kamarthi, S. (2014). Solving large scale disassembly line balancing problem with uncertainty using reinforcement learning. Journal of Intelligent Manufacturing, 25(4):647-659.

[38] Xie, W. and Ahmed, S. (2018). Distributionally robust simple integer recourse. Computational Management Science, 15:351-367.

[39] Zhang, Y., Jiang, R., and Shen, S. (2018). Ambiguous chanceconstrained binary programs under mean-covariance information. SIAM Journal on Optimization, 28(4):2922-2944.

[40] Zhang, Y., Shen, S., and Erdogan, S. A. (2017). Solving 0-1 semidefinite programs for distributionally robust allocation of surgery blocks. Optimization Letters, 12(7):1503-1521.

[41] Zhang, Z., Denton, B., and Xie, X. (2015). Branch and price for chance constrained bin packing. Available at Optimization-Online http://www.optimization-online.org/DB_HTML/2015/11/5217.html.

[42] Zitzler, E., Deb, K., and Thiele, L. (2000). Comparison of multiobjective evolutionary algorithms: Empirical results. Evolutionary computation, 8(2):173-195. 